\title{
New experimental sublimation energy measurements for some relevant astrophysical ices
}

\author{
R. Luna, M. Á. Satorre, C. Santonja, and M. Domingo
}

\begin{abstract}
Centro de Tecnologías Físicas. Universitat Politècnica de València, Plaza Ferrándiz Carbonell, 03801 Alcoy, Spain e-mail: ralunam@fis.upv.es
\end{abstract}

Received 13 December 2013 / Accepted 5 April 2014

\begin{abstract}
Context. The knowledge of the sublimation energy of ices allows us to better understand the dynamics between surfaces and atmospheres of different environments of astrophysical interest where ices are present.

Aims. This work is intended to provide sublimation energy values for a set of pure ices $\left(\mathrm{CO}, \mathrm{CH}_{4}, \mathrm{CO}_{2}, \mathrm{~N}_{2}\right.$, and $\left.\mathrm{NH}_{3}\right)$ using a new experimental procedure. The results were compared to some values obtained by other authors under different conditions and/or methods, to check the reliability of this new method.

Methods. We used the frequency variation obtained from a quartz crystal microbalance to calculate the sublimation energy from the Polany-Wigner equation for the first time.

Results. The results obtained are relevant since there are few previous values of sublimation energy reported on these molecules in these conditions of pressure and temperature, which are representative of astrophysical regions. These values are needed in models used to interpret dynamics of icy surfaces. In general, our results compare well to other ones obtained by different methods and complement those previously available.
\end{abstract}

Key words. methods: laboratory: solid state - planets and satellites: atmospheres - planets and satellites: physical evolution planets and satellites: surfaces

\section{Introduction}

In astrophysics, the study of ices is important since their physical and chemical characteristics play an important role in the dynamics of the wide range of places where they are present: in the interstellar medium (ISM), in the denser and cooler regions of molecular clouds (Whittet et al. 1996; Roberts et al. 2007; Cuppen \& Herbst 2007), and in the solar system in objects such as planets, satellites, trans-Neptunian objects (TNOs; Quirico et al. 1999; Licandro et al. 2006; Grundy et al. 2006; Cruikshank et al. 2000; Forget et al. 2005; Barucci et al. 2006), or comets (Mumma et al. 2005; A'Hearn et al. 2005).

Even though water is the most abundant ice present in astrophysical environments (Domingo 2003; and van Broekhuizen 2005), there are many relevant molecules that in some of these places dominate or deeply influence icy surfaces. Among these ices are $\mathrm{CO}, \mathrm{CH}_{4}, \mathrm{CO}_{2}, \mathrm{~N}_{2}$, and $\mathrm{NH}_{3}$. For the ISM, the presence in dense molecular clouds of solid carbon monoxide on interstellar grains was confirmed observationally in 1984 by its infrared absorption at $4.67 \mu \mathrm{m}$ (Whittet \& Duley 1991), methane was detected forming part of the ice grain mantles of young stars (Lacy et al. 1991), carbon dioxide (Gerakines et al. 1999) and nitrogen ices toward many of these clouds (Sandford et al. 2001), and solid ammonia was found as part of the interstellar ices in the line of sight of deeply embedded stellar objects such as W33A (Gurtler et al. 2002).

In the solar system: methane is present on a surface composed of an $\mathrm{N}_{2}: \mathrm{CO}: \mathrm{CH}_{4}$ terrain on Triton (Quirico et al. 1999) and on Pluto, but in both objects, $\mathrm{CH}_{4}$ could be segregated from the other molecules present on these surfaces (Lellouch et al. 2011; Douté et al. 1999). Solid nitrogen has also been observed on both Triton and Pluto as the main component of their surfaces (Cruikshank et al. 1993; Owen et al. 1993) forming a structure with other minor components, $\mathrm{CO}$ and $\mathrm{CH}_{4}$. Solid carbon dioxide is the main component of the ice present in Mars (Haberle et al. 2004; Aharonson et al. 2004), and it could also be present in the upper parts of water-ice-dominated surfaces of the Galilean satellites Callisto and Ganymede (McCord et al. 1997, 1998) owing to the action of ions coming from the magnetosphere of Jupiter. On Europa's surface, $\mathrm{CO}_{2}$ has been detected from the strong absorption band centered at $4.25 \mu \mathrm{m}$ (Hansen \& McCord 2008). The presence of $\mathrm{CO}$ is also detected in comets such as 29P/Schwassmann-Wachmann 1 (Gunnarsson et al. 2008). As for comets, the study of physical properties of TNOs is fundamental to understanding their origin and evolution. In one of the TNOs, Orcus, $\mathrm{NH}_{3}$ has also been tentatively detected (Barucci et al. 2008).

A relevant parameter for studying the dynamics of the processes that take place on icy surfaces is the sublimation energy, $E_{\text {sub. }}$. This is defined as the change in energy between the solid and gas phases of a species. Theoretically, sublimation refers to a process where the whole ice is involved; however desorption is a process where only surface molecules are involved. Nevertheless, both processes are used without distinction in the literature (see for example Sandford \& Allamandola 1993). This value can be used to estimate the sublimation rate, which is relevant for the evolution of surfaces of astrophysical objects in lowtemperature regions where ices are present (Viti et al. 2004).

Pluto's tenuous atmosphere is a benchmark example of an atmosphere controlled by vapor-pressure equilibrium with surface ices. Pluto's surface is dominated by $\mathrm{N}_{2}$ ice, but other detected surface compounds include $\mathrm{CH}_{4}$ and $\mathrm{CO}$ (Lellouch et al. 2011). 


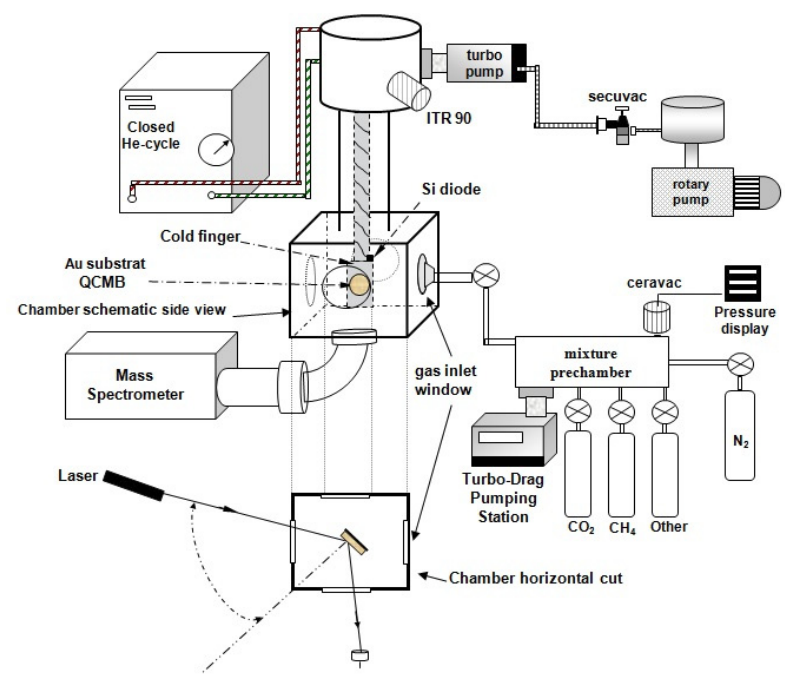

Fig. 1. Diagram of the experimental setup.

Like Pluto, Neptune's satellite and probably former Kuiper-Belt object Triton present a tenuous, predominantly nitrogen atmosphere, in equilibrium with surface ices mostly composed of $\mathrm{N}_{2}$. The most volatile of these species, $\mathrm{CH}_{4}$ and $\mathrm{CO}$, must be present in trace amounts in the atmosphere as well (Lellouch et al. 2010). Therefore, it is of interest to obtain values of sublimation energy in the laboratory for these molecules relevant in astrophysical contexts. This parameter is also useful since the course of chemical differentiation (Kargel 1991) depends very much on the sublimation energy and relative densities of important phases.

To obtain the sublimation energy we have developed a new method that uses a quartz crystal microbalance (QCMB) under high vacuum (HV) conditions. This method and its comprehensive procedure is presented and described in detail in Luna et al. (2012). Our method, based on HV conditions, permits the sublimation energy to be calculated directly from the analysis of the QCMB signal thanks to the release of the molecules from the solid phase when the substrate is warmed up in a controlled manner. This process follows a zeroth-order desorption process during the sublimation as the surface molecules sublimate, more molecules underneath and under the same conditions are exposed. The remarkable fact is that molecules sublimate directly from the QCMB. It acts simultaneously as a sample holder and detector. As far as we know, this is the sole direct method of measuring molecules' release.

In this paper we present new results for the sublimation energy for five ices related to astrophysical environments. These values can be used to better reproduce physical conditions and interactions between ices. Our results are compared to the few previously reported in the literature. This article first reviews the experimental setup. Section 3 presents a brief summary of the method we have implemented to calculate the sublimation energy and describe the other methods previously used, next the experimental results are presented and compared to results reported by other authors using different methods in Sect. 4. In Sect. 5 the discussion and astrophysical implication are shown, and finally in Sect. 6 conclusions are presented.

\section{Experimental setup}

The basic components of our experimental configuration (Fig. 1) are a high-vacuum and low-temperature system, a QCMB, a laser, and a quadrupole mass spectrometer (QMS). The vacuum in the chamber is obtained with a turbomolecular pump backed up by a rotary pump. The first stage of a closed-cycle He cryostat $(40 \mathrm{~K})$, thermally connected to a shield protector acts as a cryopump providing a base pressure below $10^{-7} \mathrm{mbar}$ (HV conditions) measured with an ITR IoniVac transmiter (5\% in accuracy). The pressure of gases is monitored by means of the QMS (AccuQuad RGA 100 with a resolution of $\sim 0.5$ amu and an ionization energy of $70 \mathrm{eV}$ ) during the whole experiment, allowing us to check the composition of the gases entering the chamber. The second stage of the cryostat $(240 \mathrm{~mm})$ and the sample holder $(70 \mathrm{~mm})$ are both referred to in this paper, as the cold finger. The QCMB is located $2 \mathrm{~cm}$ after the second stage in the sample holder, held to the cold finger by means three plastic strips, and the diode is beside it, fixed with a metal flange. This configuration reaches $12 \mathrm{~K}$ at the sample holder, in thermal contact with the QCMB (Q-Sense X 301; AT-cut; 0216/1; 5 MHz). The temperature in the sample (QCMB) is controlled by the Intelligent Temperature Controller ITC 503S (Oxford Instruments). It uses the feedback of a silicon diode sensor (scientific Instruments) located just beside the quartz, allowing the temperature to vary between 12.0 and $300.0 \pm 0.5 \mathrm{~K}$ by means a resistive heater.

Gas in the study is charged in a prechamber at a suitable pressure measured with a Ceravac CTR 90 (Leybold vacuum), whose accuracy is $0.2 \%$, provided with a ceramic sensor not influenced by the gas type (range $10^{4}$, FS 100 torr). Gas enters the chamber through a needle valve (Leybold D50968) that regulates the gas flow. The gas fills the chamber and sticking where the temperature is below the temperature of sublimation. This implies a deposition in the QCMB coming from all the parts of the chamber, which is called background deposition. This kind of deposition hampers the use of the QMS to monitor the sublimation from the QCMB because it detects any molecule that is sublimating, but not only from the QCMB. The interferometric pattern of He-Ne laser $(632.8 \mathrm{~nm})$ has been used to determine the thickness of the ice deposited. Once a film depth of around $0,5 \mu \mathrm{m}$ is reached, ice is desorbed at a constant rate of $1 \mathrm{~K} \mathrm{~min}^{-1}$, which is controlled by the ITC.

The following chemicals have been used in this research: $\mathrm{CH}_{4}-99.9995 \%$ (Praxair), $\mathrm{CO}_{2}-99.998 \%$ (Praxair), $\mathrm{N}_{2}-$ 99.999\% (Air Products), $\mathrm{NH}_{3}-99.999 \%$ (Praxair), and $\mathrm{CO}-$ 99.997\% (Praxair). To preserve gas purity, the whole prechamber is evacuated with a turbo-drag pumping station (ultimate pressure $<10^{-6}$ mbar).

\section{Experimental methods to determine sublimation energy}

This section gives a brief description of the methods used to determine the sublimation energy. Firstly, the QCMB analysis proposed by us (method 1, M1) is described. Secondly, we briefly explain the most relevant methods in the literature related to the molecules we focus on, referred to as M2, M3, and M4.

\subsection{Analysis of the $Q C M B$ frequency variation, $M 1$}

The QCMB makes use of the piezoelectric properties of a quartz crystal (Sauerbrey 1959; Lu \& Owen 1972; Benes 1984), whose frequency variation, owing to the mass change, follows the Sauerbrey equation:

$\Delta f_{0}=-S \cdot \Delta m$.

In this equation, $f_{0}$ is the resonant frequency of the crystal, and $S$ the Sauerbrey constant. The variation in frequency $\Delta f_{0}$ is caused 
R. Luna et al.: New experimental sublimation energy measurements for some relevant astrophysical ices

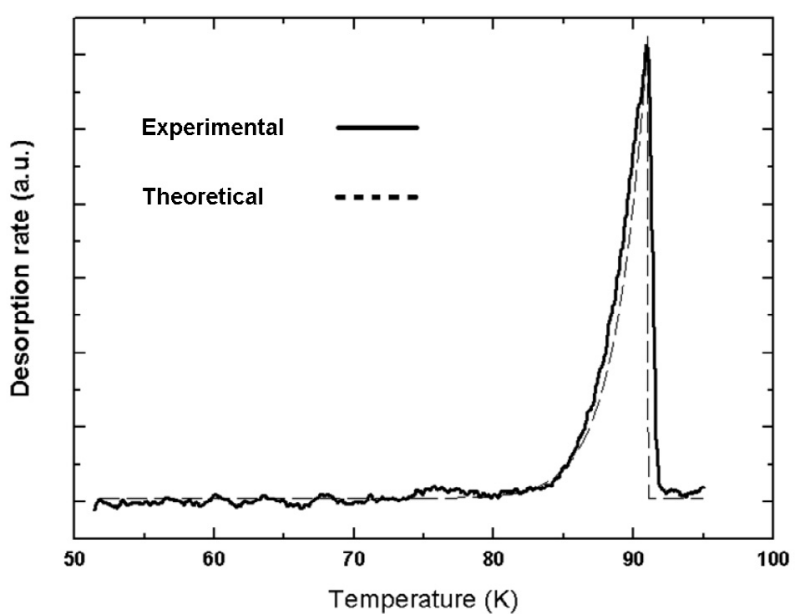

Fig. 2. Desorption curves corresponding to processes of zeroth-order kinetics: theoretical (dashed line) and derivative versus time of the QCMB experimental signal (solid line) for $\mathrm{CO}_{2}$.

by the accretion or desorption of material $\Delta m$ between the gas phase and the substrate.

To obtain the sublimation energy of pure ices, experiments of desorption at a constant rate of warming up have been carried out. As molecules sublimate, the oscillation frequency increases. From Eq. (1), the desorption rate can be calculated as the derivative of the frequency versus time, which is used to determine the sublimation energy. The profile of the desorption rate depends on the kinetics of the process. Our results fit a zeroth-order desorption characterized by an increase in desorption rate exponentially with temperature, and a rapid drop after the maximum desorption rate. To fulfill these conditions as accurately as possible, we do not exceed an ice depth of $0.5 \mu \mathrm{m}$ during deposition (to avoid relevant changes in the outer layers of the film, even the "breaking" of the ice), and the last monolayers desorbed (below $~ 1 \mathrm{~nm}$ ) should be ignored since these molecules could be affected by interactions with the surface of the balance.

These features are shown in Fig. 2, where the theoretical and our experimental desorption for $\mathrm{CO}_{2}$ are shown. These kinetics can be modeled by a process described by the Polanyi-Wigner equation:

$r_{\mathrm{des}}=-A \cdot \exp \left(-\frac{E_{\mathrm{sub}}}{R T}\right) n^{0}$

where $r_{\text {des }}$ is the desorption rate, $A$ the preexponential sublimation factor, $R$ the constant for ideal gases, $T$ the absolute temperature, $E_{\text {sub }}$ the activation energy for sublimation, and $n$ the number of molecules on the surface. This kinetics is essentially independent of the coverage; i.e., as the surface molecules sublimate, more molecules underneath, under the same conditions are exposed. Consequently, the number of molecules in the interphase film-air remains constant.

To proceed with the overall analysis of the QCMB, it is necessary to take two contributions to its signal into account: the continuous deposition of contaminants (mainly water) and the effect of the temperature in the QCMB signal. Firstly, to quantify and remove contaminants we follow the following procedure. We take the frequency signal at a certain strategic temperature at two different instants, one during the cooling down and the other during the warming up. The temperature is selected at a point slightly above the sublimation temperature ( $\left.T_{\text {removal }}\right)$. Even though the frequency signal should coincide (because is the same temperature) once the ice is completely sublimated, there is a shift in frequency for these two instants due to the amount of contaminants deposited during the experiment. Assuming a constant rate of contamination (since its pressure remains constant during the whole experiment), it is possible to subtract it during the experiment. Secondly, the temperature influence on the balance signal is corrected. A linear relationship between frequency and temperature characterizes our system. This relationship is obtained within the range of temperatures between the temperature referred to above $\left(T_{\text {removal }}\right)$ and the onset of the accretion process. Once these contributions are calculated and removed, we obtain a QCMB signal that only depends on the material deposited on its surface. The correction procedure is explained in detail in Luna et al. (2012).

Figure 2 represents the experimental desorption for $\mathrm{CO}_{2}$. In this plot the desorption rate is calculated as the derivative of the experimental frequency obtained from the QCMB signal and represented against temperature. The sharp, asymmetric peak, localized around $92 \mathrm{~K}$, indicates the highest sublimation rate. Comparing the profile of this curve with the theoretical one shown in Fig. 2 we can assume a zeroth-order desorption kinetics to use the Polanyi-Wigner equation (Eq. (2)) in our sublimation experiments. Assuming this zeroth-order desorption, our results are repeatable within $0.5 \mathrm{~K}$ in our experimental conditions.

The energy of sublimation is obtained from the slope of the fit to a straight line of the $\ln \left(r_{\text {des }}\right)$ versus $1 / T$. Our experiments have been performed several times for each molecule to check the repeatability of the procedure.

\subsection{Analysis of the vapor pressure of the solid phase versus temperature, M2}

These experiments (Armstrong et al. 1955; Jones 1960; Stephenson \& Malanowski 1987) use an experimental apparatus based in an evacuated vessel surrounded by a bath of liquid air in a Dewar flask whose temperature is given by a platinum resistance thermometer. The vacuum in the vessel is produced by a vacuum pump, and the final pressure is measured with a differential oil-manometer ranging pressures from $10^{-1}$ to $10^{3}$ mbar. The variation in the sublimation pressure with temperature may be represented as an approximation to the Clausius-Clapeyron equation by $\operatorname{Ln}(p)=B-E_{\mathrm{sub}} /(R T)$, where $p$ is the pressure of the saturated vapor at the absolute temperature $T, B$ is a constant, and $R$ is the gas constant. Therefore representing $\operatorname{Ln}(p)$ versus $1 / T$ the slope of the straight line obtained is $E_{\text {sub }} / \mathrm{R}$.

This method has been used under conditions not related to astrophysical regions since its experimental setup does not allow reaching lower temperatures and pressures; nevertheless, we have considered these results of interest since it allows comparing sublimation energy in very different experimental conditions. For this $\mathrm{M} 2$, only results for $\mathrm{CH}_{4}, \mathrm{CO}_{2}$, and $\mathrm{CO}$ have been reported, and there is only one published result for the last two molecules. There are no results for $\mathrm{NH}_{3}$ and $\mathrm{N}_{2}$.

\subsection{Analysis of the infrared absorption band strength at different temperatures close to the sublimation temperature, $M 3$}

These more recent experiments (Sandford \& Allamandola 1988, 1990a,b, 1993) were performed under much lower pressure conditions. The experimental apparatus uses a closed-cycle helium refrigerator to cool down the system, an HV chamber to obtain pressures lower than $5 \times 10^{-8}$ mbar, and infrared spectroscopy. 
In this method, several temperatures below the sublimation one of the substance under study are chosen, and the sublimation energy is derived from the desorption rate at these temperatures. For each temperature selected, authors calculate the rate of desorption from the decrease in the band strength versus time, and the energy of sublimation (in K) is obtained by using Eq. (3). To check the result, this procedure is used at different temperatures, and the sublimation energy is calculated for each one. This procedure can only be performed for temperatures below the sublimation one, but the closer the temperature is to the sublimation one, the faster the process occurs. If the selected temperature is more than $10 \mathrm{~K}$ (from the desorption temperature), the measurements could last several days (or even years), so two or three temperatures are selected just below the sublimation one, and a mean value for sublimation energy is obtained. The procedure for determining $E_{\text {sub }}$ is based on the models of Langmuir (1916) and Frenkel (1924) which assume that the sticking efficiency is equal to 1.0 , leading to the expression

$\frac{E_{\text {sub }}}{k}=-T \ln \left[\frac{a_{\mathrm{m}}}{v_{0} t_{\mathrm{f}} A}\left\{(\tau \Delta v)_{\mathrm{i}}-(\tau \Delta v)_{\mathrm{f}}\right\}\right]$,

where $E_{\text {sub }}$ is the sublimation energy, $k$ the Boltzman constant, $T$ the absolute temperature, $a_{m}$ the area of a molecular site of the species, $v_{0}$ the lattice vibrational frequency of the molecule under study in the solid matrix, $t_{f}$ the time over which the loss rate is measured, $(\tau \Delta v)_{i}$ the integrated band strength at time $\mathrm{t}_{i}=0$, $(\tau \Delta v)_{f}$ the integrated band strength at time $\mathrm{t}_{f}$, and $A$ the integrated absorbance of the solid under study in $\mathrm{cm}$ molecule ${ }^{-1}$. The experiment is performed several times at different temperatures. For each experiment, an $E_{\text {sub }}$ is calculated, and the average of all the results is obtained.

Since the integrated absorbance is used in this method, it is necessary to use as input the density of the ice. This value is assumed to be $1 \mathrm{~g} \mathrm{~cm}^{-3}$; however, it has been demonstrated in recent publications (Satorre et al. 2008) that this value could be under- or overestimated, and as a consequence the integrated absorbance could be influenced.

\subsection{Analysis of the mass spectroscopy signal for a temperature programmed desorption (TPD) experiment, M4}

In temperature programmed desorption (TPD) method, the experiments (Acharyya et al. 2007; Bolina et al. 2005; Bischop et al. 2006; and Muñoz-Caro et al. 2010) are performed using ultra high vacuum (UHV) conditions (pressure $\leq 10^{-10} \mathrm{mbar}$ ). The necessary experimental apparatus is similar to the previous method, but for this one, mass spectroscopy is used instead.

The model is based on fitting the pressure curve obtained during desorption to a Polany-Wigner equation:

$R_{\mathrm{des}}=\frac{\mathrm{d} N_{g}}{\mathrm{~d} t}=v_{i}\left[N_{\mathrm{s}}\right]^{i} \exp \left[-\frac{E_{\mathrm{sub}}}{T}\right]$,

where $R_{\text {des }}$ is the desorption rate, $N_{g}$ the number of molecules desorbing from the substrate, $v_{i}$ a pre-exponential factor for desorption order $i, N_{\mathrm{s}}$ the number density of molecules on the surface at a given time t, and $E_{\text {sub }}$ the sublimation energy (in K).

In this method, the presence of the desorbing molecules is measured in the vacuum chamber by means of a mass spectrometer separated from the sample holder where the ice is grown, then it is assumed that the molecules released are instantaneously detected by the spectrometer. In our method, the release of molecules is directly measured from the sample holder,

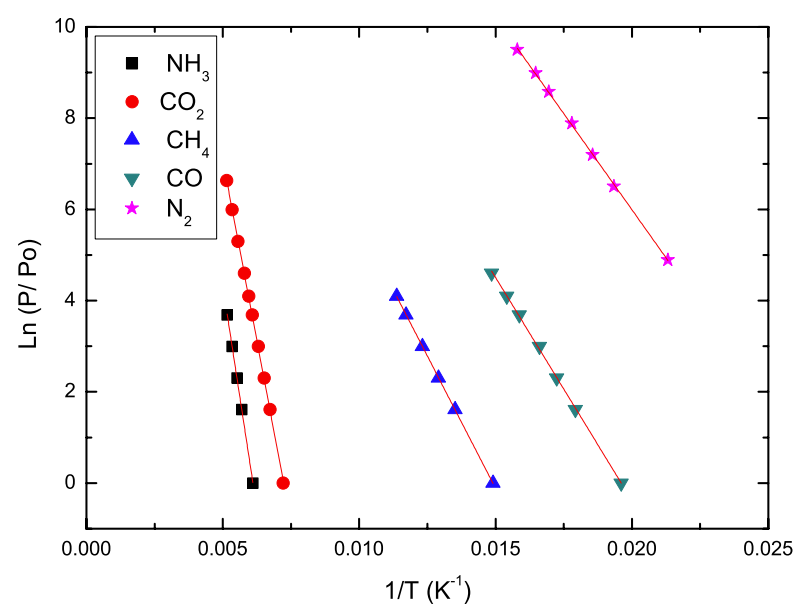

Fig. 3. $\operatorname{Ln}(P / P o)$ versus $1 / T$ for the five molecules under study. The values of pressure and temperature have been taken from the database of Stull 1947. The pressure reference $P o$ is taken as 1 bar. Calculated solid lines represent the fitting performed for each substance to obtain the sublimation energy (Method M2 described in the text).

since the QCMB acts at the same time as a sample holder and as a detector.

\section{Results}

A set of desorption experiments was performed to provide the sublimation energy for some ices for which few data have previously been published. Additionally, since the values of sublimation energy reported so far have been obtained by different methods, it is possible to compare our results with the other ones in order to check the validity of our instrument. For this purpose, we divided the results available in the literature and our results into four groups corresponding to the methods explained above:

- Method 1 (M1) is formed by our experimental results obtained from the frequency variation of a QCMB.

- Method 2 (M2) comprises results obtained by the analysis of the variation in the saturated vapor pressure of the solid phase versus temperature.

Because of the lack of data for $\mathrm{NH}_{3}$ and $\mathrm{N}_{2}$ ices obtained with method 2, we calculated new energy sublimation values by using the Stull database (Stull 1947) of pressure and temperature for these two molecules and the other ones under study. From this database, we only took the values of pressure and temperature below the triple point for each molecule. Figure 3 shows the plots of the $\operatorname{Ln}(p)$ versus $1 / T$ performed to obtain $E_{\text {sub }}$ from M2. The statistics of the fit, along with the values of the $E_{\text {sub }}$, are compiled in Table 1 . This table also reports the temperature and pressure of the triple point for each molecule and the interval of temperatures and pressures selected from the work of Stull to carry out this study. A correlation coefficient higher than 0.999 is obtained for all the ices.

- Method 3 (M3) incorporates a collection of experiments in which the sublimation energy is obtained based on the sublimation rate of an ice at different temperatures close to the sublimation temperature of this ice. The desorption rate is measured by means of infrared spectroscopy.

- Method 4 (M4) compiles values obtained from TPD experiments and a further fit of a Polany-Wigner equation to reproduce the experimental desorption curve. In this case the desorption rate is monitored by mass spectroscopy. 
R. Luna et al.: New experimental sublimation energy measurements for some relevant astrophysical ices

Table 1. Triple point values and sublimation energies calculated using database reported in the work of Stull (1947).

\begin{tabular}{lccl|cccc}
\hline \hline Substance & $T_{t}(\mathrm{~K})$ & $P_{t}(\mathrm{bar})$ & Reference & $T$. range $(\mathrm{K})$ & $P$. range $($ bar $)$ & $R$ & $E_{\text {sub }}\left(\mathrm{kJ} \mathrm{mol} \mathrm{m}^{-1}\right)$ \\
\hline Carbon dioxide & 216.58 & 5.185 & Span and Wagner (1996) & $138.7-194.8$ & $0.001-1.013$ & 0.9997 & $26.4 \pm 0.2$ \\
Methane & 90.68 & 0.1169 & Friend et al. (1989) & $67.1-87.9$ & $0.001-0.080$ & 0.9998 & $9.64 \pm 0.02$ \\
Carbon monoxide & 68.12 & 0.1540 & Goodwin (1985) & $51.0-67.3$ & $0.001-0.133$ & 0.9995 & $8.13 \pm 0.09$ \\
Ammonia & 195.4 & 0.0601 & Xiang (2004) & $163.9-193.8$ & $0.001-0.053$ & 0.9998 & $32.6 \pm 0.2$ \\
Molecular nitrogen & 63.14 & 0.1252 & Jacobsen et al. (1986) & $46.9-63.3$ & $0.001-0.133$ & 0.9997 & $7.01 \pm 0.06$ \\
\hline
\end{tabular}

Notes. $R$ represents the correlation coefficient obtained for the linear fit.

Table 2. Compilation of sublimation energies for the ices under study, obtained by different methods.

\begin{tabular}{|c|c|c|c|c|c|c|}
\hline Substance & $T(\mathrm{~K})$ & $E_{\mathrm{sub}}\left(\mathrm{kJ} \mathrm{mol}^{-1}\right)$ & $E_{\text {sub }}(\mathrm{K})$ & Method & References & $\bar{E}_{\mathrm{sub}}\left(\mathrm{kJ} \mathrm{mol}^{-1}\right)$ \\
\hline Carbon & $91.5-92.5$ & $29.3 \pm 0.3$ & $3530 \pm 36$ & 1 & This work & \multirow{4}{*}{$26 \pm 3$} \\
\hline \multirow[t]{3}{*}{ dioxide } & $138.7-194.8$ & $26.4 \pm 0.2$ & $3162 \pm 24$ & 2 & Calculated, based on Stull (1947) & \\
\hline & $198-216$ & 26.1 & 3139 & 2 & Stephenson \& Malanowski (1987) & \\
\hline & 80-90 & 22.37 & 2690 & 3 & Sandford \& Allamandola (1990b) & \\
\hline \multirow[t]{5}{*}{ Methane } & $35.5-36.5$ & $8.5 \pm 0.3$ & $1000 \pm 35$ & 1 & This work & \multirow{5}{*}{$9.4 \pm 0.7$} \\
\hline & $67.1-87.9$ & $9.64 \pm 0.02$ & $1157 \pm 2$ & 2 & Calculated, based on Stull (1947) & \\
\hline & 54-90 & 9.2 & 1106 & 2 & Armstrong et al. (1955) & \\
\hline & $79-89$ & 10.0 & 1203 & 2 & Jones $(1960)$ & \\
\hline & $53-91$ & 9.7 & 1166 & 2 & Stephenson \& Malanowski (1987) & \\
\hline \multirow{7}{*}{$\begin{array}{l}\text { Carbon } \\
\text { monoxide }\end{array}$} & $33.5-34.5$ & $6.3 \pm 0.2$ & $760 \pm 24$ & 1 & This work & \multirow{7}{*}{$7.3 \pm 0.6$} \\
\hline & $51.0-67.3$ & $8.13 \pm 0.09$ & $974 \pm 11$ & 2 & Calculated, based on Stull (1947) & \\
\hline & $54-61$ & 7.6 & 914 & 2 & Stephenson \& Malanowski (1987) & \\
\hline & $40-50$ & 7.98 & 960 & 3 & Sandford \& Allamandola (1990a) & \\
\hline & $28.5-29.5$ & 7.11 & 855 & 4 & Bisschop et al. (2006) & \\
\hline & $28.5-29.5$ & 7.13 & 858 & 4 & Acharyya et al. (2007) & \\
\hline & $28-29$ & 6.94 & 834 & 4 & Muñoz-Caro et al. (2010) & \\
\hline \multirow[t]{4}{*}{ Ammonia } & $112.5-113.5$ & $31.8 \pm 0.5$ & $3830 \pm 60$ & 1 & This work & \multirow{4}{*}{$28 \pm 4$} \\
\hline & $163.9-193.8$ & $32.6 \pm 0.2$ & $3926 \pm 24$ & 2 & Calculated, based on Stull (1947) & \\
\hline & $100-105$ & 25.57 & 3075 & 3 & Sandford \& Allamandola (1993) & \\
\hline & $100-108$ & 23.15 & 2784 & 4 & Bolina \& Brown (2005) & \\
\hline \multirow{3}{*}{$\begin{array}{l}\text { Molecular } \\
\text { nitrogen }\end{array}$} & $24.5-25.5$ & $4.3 \pm 0.2$ & $520 \pm 24$ & 1 & This work & \multirow{3}{*}{$6.0 \pm 1.7$} \\
\hline & $46.9-63.3$ & $7.01 \pm 0.06$ & $843 \pm 7$ & 2 & Calculated, based on Stull (1947) & \\
\hline & $26-27$ & 6.65 & 800 & 4 & Bisschop et al. (2006) & \\
\hline
\end{tabular}

One of the goals of this paper is to compare different values of sublimation energy coming from several methods. To facilitate this task, we plot the sublimation energy versus temperature for each molecule (Figs. 4 and 5). Since temperature is not constant during any desorption experiment and since every method uses a different procedure, in these plots the meaning of the abscissas is different for each method. For method M1, $T$ is the temperature at which the desorption rate reaches the maximum. For $\mathrm{M} 2, T$ is the mean temperature for the interval in which the vapor-saturated pressure has been analyzed. For M3, $T$ is the mean temperature taken to obtain the sublimation rate. For M4, $T$ is the temperature taken (directly from the plot reported in the corresponding paper) as the average of the peak of desorption temperatures for different experiments performed with different coverages. From our definition of $T$ for each method, this parameter is an interval rather than a point, in our experimental results (M1) T represents the narrowest interval $(\leq 0.5 \mathrm{~K})$, followed by M4 ( $\leq 1 \mathrm{~K})$, for M3 the interval is $\leq 5 \mathrm{~K}$, and for method M2 this temperature can even represent an interval greater than $50 \mathrm{~K}$.

The results obtained by all the methods are reported in Table 2, where we present sublimation energy values (Col. 3) and their corresponding interval of temperatures (Col. 2) considered to obtain the sublimation temperature. Column 4 shows sublimation energy expressed in $\mathrm{K}$ (obtained as $E_{\text {sub }} / k N_{A}$ ), in order to ease the comparison to some results obtained by other authors and reported using these units. Column 5 represents the corresponding method previously defined, and Col. 7 shows the average of the values obtained for different methods for each substance and its corresponding standard deviation.

The results are also plotted in Figs. 4 and 5. To better interpret the results, five panels representing the five molecules under study present the same scale in abscissas and ordinates. In these plots, sublimation energy versus sublimation temperature is represented for $\mathrm{CO}_{2}$ (Fig. 4, upper panel), $\mathrm{CH}_{4}$ (Fig. 4, intermediate panel), $\mathrm{CO}$ (Fig. 4, lower panel), $\mathrm{NH}_{3}$ (Fig. 5, upper panel), and $\mathrm{N}_{2}$ (Fig. 5, lower panel). In abscissas the interval of temperatures reported in Table 2 is represented and the sublimation energy value with the corresponding error (when known) appears in ordinates.

As an overall result, we can observe for all the molecules we study that different methods lead to similar values, giving validity to our experimental procedure. The highest deviation, when comparing our results to the others, is the case of nitrogen, where our result is significantly lower than the other two. In general, a slight tendency to increase sublimation energy with temperature seems to be detected for these molecules, but the lack of error bars for the results taken from the literature does not allow this tendency to be confirmed.

\section{Discussion and astrophysical implications}

The results obtained here are directly applicable to those astrophysical environments where molecules grow separately as pure molecules. In many of these places the molecules studied in this 

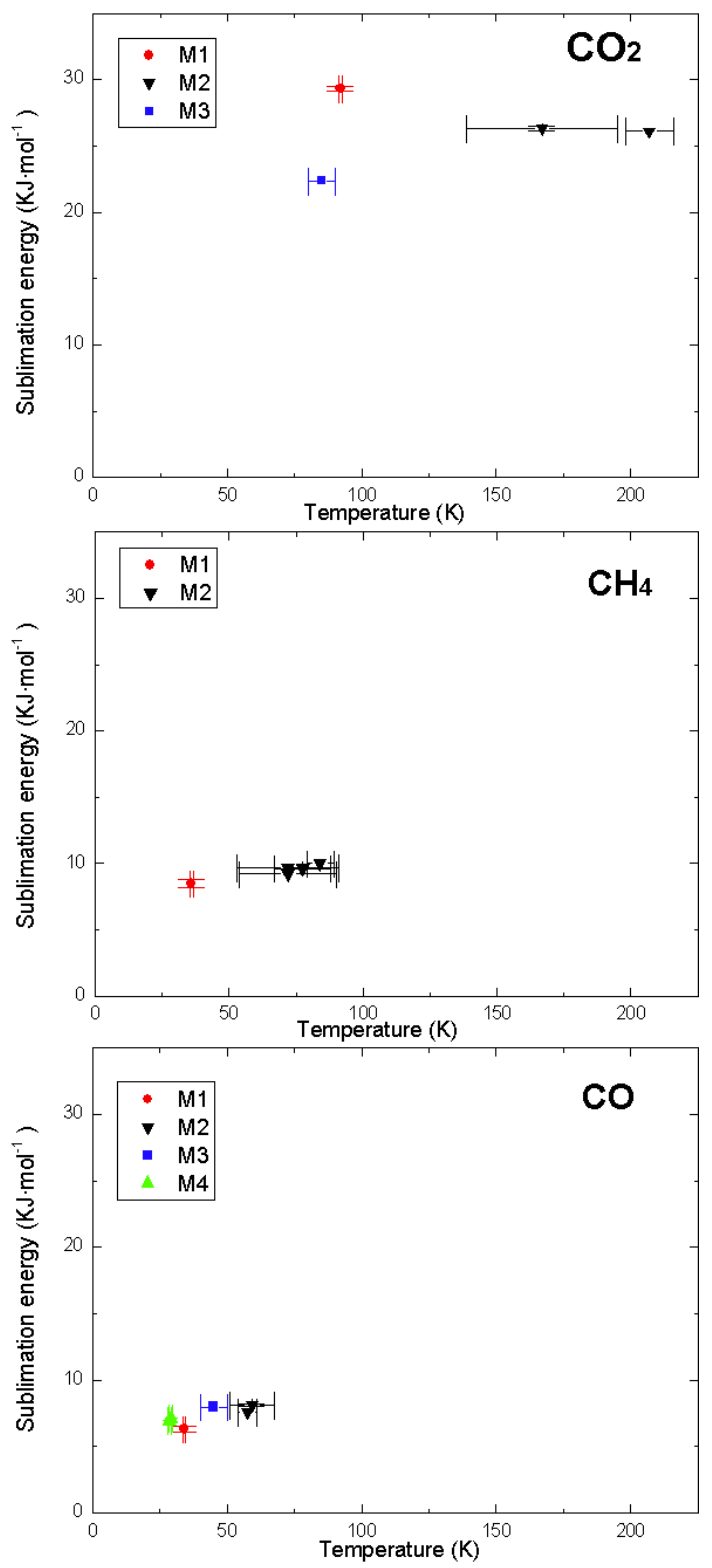

Fig. 4. Comparison of sublimation energy obtained by different methods for $\mathrm{CO}_{2}, \mathrm{CH}_{4}$, and $\mathrm{CO}$. In all plots our experiments (M1) are represented by solid circles. Inverted triangles represent the experiments measured by M2, squares represent results reported by M3, and triangles those obtained by M4.

work can be trapped in an $\mathrm{H}_{2} \mathrm{O}$ matrix. In this sense, Collings et al. (2004) categorize different molecules depending on their desorption process in three groups: $\mathrm{CO}$-like, $\mathrm{H}_{2} \mathrm{O}$-like, and intermediate. The molecules studied in our work belonging to the first group are $\mathrm{CO}, \mathrm{N}_{2}$, and $\mathrm{CH}_{4} . \mathrm{NH}_{3}$ lies in the second group, and as intermediate there is $\mathrm{CO}_{2}$. Despite the Collings et al. (2004) work with mixtures of all these molecules with water ice in different deposition conditions, the behavior of each particular pure ice is studied for every case to identify the effect of water ice on it. Then the behavior of pure molecules help for understanding the effect of mixtures on a particular property. Experimental results can be used as input for models, as is the case of Viti et al. (2004) with the data of Collings et al. (2004). Our results would help those computational and experimental groups working with both pure and mixed ices.
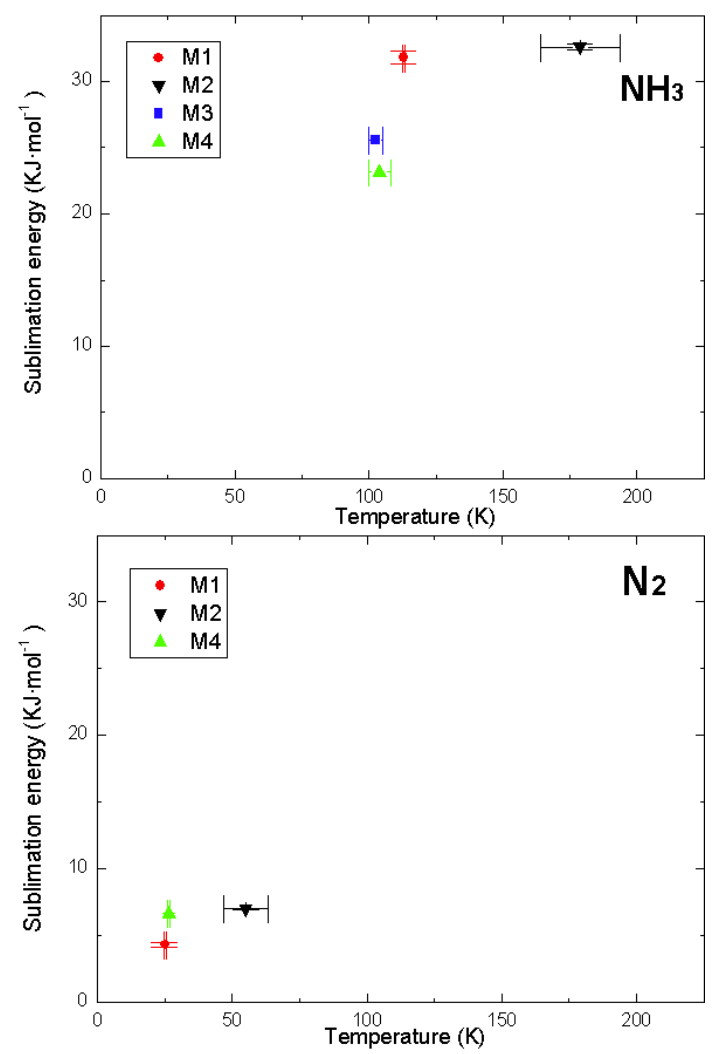

Fig. 5. Comparison of sublimation energy obtained by different methods for $\mathrm{NH}_{3}$ and $\mathrm{N}_{2}$. In all plots our experiments (M1) are represented by solid circles. Inverted triangles represent the experiments measured by $\mathrm{M} 2$, squares represent results reported by M3, and triangles those obtained by M4.

Energy of desorption is a parameter that allows the binding energy of molecules in the solid phase to be evaluated. This parameter is very important and put in evidence the intriguing question exposed by Sandford \& Allamandola (1993) when they applied the calculated energy to the ISM: when taking the binding energy of the ices studied and the residence time in gas phase into consideration, all the molecules should be depleted from the gas phase onto the cold grain surface in the dense ISM grains, in the absence of other effects than the thermal one. To help understand how it can be possible to observe those molecules in the gas phase in many lines of sight, the effect of irradiation on ices has been studied. Muñoz-Caro et al. (2010) demonstrate that if the effect of UV photons (induced by cosmic rays) on CO ices is taken into account, an equilibrium between solid and gas phase is reached, because of ice desorption from dust produced by secondary photon irradiation. But this is not the only interaction producing desorption of icy components. Seperuelo Duarte et al. (2010) perform a series of experiments where the desorption rate induced by $\mathrm{H}, \mathrm{Ni}$ and $\mathrm{Fe}$ ions are estimated, concluding that the effect of heavy ions is even more important than the effect of protons in the ISM. The works performed by Muñoz-Caro et al. (2010) and Seperuelo Duarte et al. (2010), are relevant for the ISM because, even though CO can be mixed with water ice, from the absorption profile studied by Ehrenfreund et al. (1996), it is possible to deduce that $\mathrm{CO}$ could be embedded in a nearly separate ice phase.

The same kind of experiment has been carried out for ammonia. The interest of ammonia and their mixtures are relevant not only for the ISM, since it is abundant up to $15 \%$ relative to water ice (Bordalo et al. 2013), but also for the outer solar system as 
can be seen for example in Moore et al. (2007). Then, as for the other molecules, the effect on desorption by heavy ions (Pilling et al. 2010) and of UV photons has been also studied (Loeffler \& Baragiola 2010).

Our results can also be applied directly to the solar system in those regions where no mixed ices appear. For example, the intriguing surface of Neptune's satellite Triton presents evidence of four of the molecules studied in the present work $\mathrm{N}_{2}, \mathrm{CO}$, $\mathrm{CH}_{4}$, and $\mathrm{CO}_{2}$. Our results are applicable for all these molecules but $\mathrm{CO}$. This is the case for nitrogen, because it is the major component of the icy surface and can be considered as pure ice. Evidence shows that $\mathrm{CO}$ and $\mathrm{CH}_{4}$ remain trapped on the nitrogen matrix, but some pure methane appears in a separate terrain. Lellouch et al. (2010) show that methane and carbon monoxide atmosphere abundances are compatible with the presence of isolated methane on the surface but not for CO. This conclusion is also reached by Grundy et al. (2010) based on observations. Then our results can be applied to that zone on the surface where pure methane is present. This is also the case for $\mathrm{CO}_{2}$ as it probably appears in a separate terrain (Cruikshank et al. 1993), although $\mathrm{CO}_{2}$ could also be present mixed with water. Considering that Triton is affected by ions present in the magnetosphere of Neptune, the same kind of calculus made for ices as $\mathrm{CO}$ and $\mathrm{NH}_{3}$, can be done for $\mathrm{N}_{2}, \mathrm{CO}_{2}$, and $\mathrm{CH}_{4}$ on the Triton surface.

Knowledge of the physical characteristics of these pure ices can help for understanding how their behavior is modified if they are mixed with water ice. This could be the case in some comets, if we take the data from the encounter of the Deep Impact mission with the comet 9P/Temple 1, since the impact was followed by the sublimation of different molecules (see for example Mumma et al. 2005). In all cases, water is the major component, and $\mathrm{CO}$ is the second most abundant molecule detected with an abundance about the $4 \%$ respect water. $\mathrm{CH}_{4}$ and $\mathrm{CO}_{2}$ also appearing in lower proportions.

As explained, the sublimation energy is a parameter widely used in astrophysics. If one knows the sublimation energy for a particular ice, its desorption rate under astrophysical conditions can be inferred. This is relevant to estimating either the residence time in a specific surface of astrophysical interest at a certain temperature (Sandford \& Allamandola 1993) or the gas composition for a specific temperature in a typical frozen surface present in planets, satellites, comets, or on the refractory surface of interstellar grains (Muñoz-Caro et al. 2010).

From the sublimation energy, it is also possible to deduce the sublimation order of the kinetics in a particular process. Bisschop et al. (2006) show how the kinetics of CO desorption is zeroth order rather than first order in cold prestellar cores and in protostars that start to heat their surroundings. Besides we have to take into account that maybe there are astrophysical situations where the desorption kinetics can be of a fractionary order rather than being always an integer, for example, a number among 0 and 1 for $\mathrm{NH}_{3}$ adsorbed onto a specific surface as demonstrated by Bolina \& Brown (2005).

\section{Conclusions}

In this paper we have obtained the sublimation energy for a set of ices at temperatures low enough to relate them to astrophysical environments as found in the ISM mantle grains, planetary icy surfaces, etc.

Our experiments are based on the variation in the frequency signal in a QCMB when an ice is desorbing from the surface of the substrate after multilayer deposition. We assume a zerothorder kinetics, which is supported when comparing our experimental desorption rate curve (see Fig. 2, solid line) to the theoretical one (Fig. 2, dashed line).

We compared our results with other ones coming from different methods in order to validate our method. In the literature, there are not many values for sublimation energy for temperatures related to astrophysical regions, so we compared our results with those reported so far, including some results coming from experiments performed at higher temperatures. We divided all the data under study into four groups depending on the method used to calculate the sublimation energy. Additionally, we complemented the results reported in the literature by calculating the sublimation energy from the database of Stull (1947) for all the molecules under study.

The results for the sublimation energy obtained for every single molecule using the different methods present standard deviations lower than $18 \%$ (except in the case of nitrogen, for which only two datapoints are found in the literature). This dispersion can be due to different assumption made for each model: M3 assumes a value for density equal to $1 \mathrm{~g} \mathrm{~cm}^{-3}$, irrespective of the ice under study; M4 measures the pressure of the gas phase of the molecule after sublimation. However, using our experimental setup, we are directly measuring the molecules released from the substrate, making it possible to perform the experiments under HV conditions. Nevertheless, we can conclude that, in general, there is good agreement among different methods for each molecule, which validates our experimental apparatus and procedure.

In summary, the results for sublimation energies will help understand the solid-gas phase composition better in many astrophysical situations. The method presented here compares well with earlier ones, but offer the advantage that the rate of desorption is directly measured from the sensor signal. Finally this method can be applied to both HV and UHV systems.

Acknowledgements. This work was supported by the Spanish Ministerio de Educación y Ciencia (Cofinanced by FEDER funds) AYA 2009-12974.

\section{References}

Acharyya, K., Fuchs, G. W., Fraser, H. J., van Dishoeck, E. F., \& Linnartz, H. 2007, A\&A, 466, 1005

A'Hearn, M. F., Belton, M. J. S., Delamere, W. A., et al. 2005, Science, 310, 258 Aharonson, O., Zuber, M. T., Smith, D. E., et al. 2004, J. Geophys. Res., 109, E05004 1

Armstrong, G. T., Brickwedde, F. G., \& Scott, R. B. 1955, J. Res. Natl. Bur. Std., 55,39

Barucci, M. A., Merlin, F., Dotto, E., Doressoundiram, A., \& de Bergh, C. 2006 , A\&A, 455, 725

Barucci, M. A., Merlin, F., Guilbert, A., et al. 2008, A\&A, 479, L13

Benes, E. 1984, J. Appl. Phys., 56, 608

Bisschop, S. E., Fraser, H. J., Öberg, K. I., van Dishoeck, E. F., \& Schlemmer, S. 2006, A\&A, 449, 1297

Bolina, A. S., \& Brown, W. A. 2005, Surface Sci., 598, 45

Bordalo, V., da Silveira, E. F., Lv, X. Y., et al. 2013, ApJ, 774, 105

Collings, M. P., Anderson, M. A., Chen, R., et al. 2004, MNRAS, 354, 1133

Cruikshank, D. P., Roush, T. L., Owen, T. C., et al. 1993, Science, 261, 742

Cruikshank, D. P., Schmitt, B., Roush, T. L., et al. 2000, Icarus, 147, 1, 309

Cuppen, H. M., \& Herbst, E. 2007, ApJ, 668, 1, 294

Douté, S., Schmitt, B., Quirico, E., et al. 1999, Icarus, 142, 421

Domingo, M. 2003, Thesis, The Politechnic University of Valencia, Spain

Ehrenfreund, P., Boogert, A. C. A., Gerakines, P. A., et al. 1996, A\&A, 315, L341

Forget, F., Levrard, B., Montmessin, F., et al. 2005, Lunar and Planetary Science, XXXVI, 1605

Frenkel, Z. 1924, Z. Phys., 26, 117

Friend, D. G., Ely, J. F., \& Ingham, H. 1989, J. Phys. Chem. Ref. Data, 18, 2, 583

Gerakines, P. A., Whittet, D. C. B., Ehrenfreund, P., et al. 1999, ApJ, 522, 357 
Goodwin, R. D. 1985, J. Phys. Chem. Ref. Data, 14, 4

Grundy, W. M., Young, L. A., Spencer, J. R., et al. 2006, Icarus, 184, 543

Grundy, W. M., Young, L. A., Stansberry, J. A., et al. 2010, Icarus, 205, 594

Gunnarsson, M., Bockelée-Morvan, D., Biver, N., Crovisier, J., \& Rickman, H. 2008, A\&A, 488, 2, 537

Gürtler, J., Klaas, U., Henning, Th., et al. 2002, A\&A, 390, 1075

Haberle, R. M., Mattingly, B., \& Titus, T. N. 2004, Geophys. Res. Lett., 31, L05702

Hansen, G. B., \& McCord, T. B. 2008, Geophys. Res. Lett., 35, L01202

Jacobsen, R. T., Stewart, R. B., \& Jahangiri, M. 1986, J. Phys. Chem. Ref. Data, 15,735

Jones, A. H. 1960, J. Chem. Eng. Data, 5, 196

Kargel, J. S. 1991, Icarus, 94, 368

Lacy, J. H., Carr, J. S., Evans, N. J. II, et al. 1991, ApJ, 376, 556

Langmuir, I. 1916, Phys. Rev., 8, 149

Lellouch, E., de Bergh, C., Sicardy, B., Ferron S., \& Käufl, H. U. 2010, A\&A, 512, L8

Lellouch, E., de Bergh, C., Sicardy, B., Käufl, H. U., \& Smette, A. 2011, A\&A, 530, L4

Licandro, J., Grundy, W. M., Pinilla-Alonso, N., \& Leisy, P. 2006, A\&A, 458, L5

Loeffler, M. J., \& Baragiola, R. A. 2010, J. Chem. Phys., 133, 214506

Lu, C., \& Owen, O. 1972, J. Appl. Phys., 43, 4385

Luna, R., Millán, C., Domingo, M., Satorre, M. Á., \& Santonja, C. 2012, Vacuum, 86, 12, 1969

McCord, T. B., Carlson, R. W., Smythe, W. D., et al. 1997, Science, 278, 271

McCord, T. B., Hansen, G. B., Clark, R. N., et al. 1998, J. Geophys. Res., 103, 8603

Moore, M. H., Ferrante, R. F., Hudson, R. L., \& Stone, J. N. 2007, Icarus, 190, 260
Mumma, M. J., DiSanti, M. A., Magee-Sauer, K., et al. 2005, Science, 310, 270

Muñoz-Caro, G. M., Jiménez-Escobar, A., Martín-Gago, J., et al. 2010, A\&A, 522, A108

Owen, T. C., Roush, T. L., Cruikshank, D. P., et al. 1993, Science, 261, 745

Pilling, S., Sepereulo-Duarte, E., Silveira, E. F., et al. 2010, A\&A, 509, A87

Quirico, E., Douté, S., Schmitt, B., et al. 1999, Icarus, 139, 159

Roberts, J. F., Rawlings, J. M. C., Viti, S., \& Williams, D. A. 2007, MNRAS, 382,733

Sandford, S. A., \& Allamandola, L. J. 1988, Icarus, 76, 201

Sandford, S. A., \& Allamandola, L. J. 1990a, Icarus, 87, 188

Sandford, S. A., \& Allamandola, L. J. 1990b, ApJ, 355, 357

Sandford, S. A., \& Allamandola, L. J. 1993, ApJ, 417, 815

Sandford, S. A., Bernstein, M. P., Allamandola, L. J., Goorvitch, D., \& Teixeira, T. C. V. S. 2001, ApJ, 548, 836

Satorre, M. Á., Domingo, M., Millán, C., et al. 2008, Planet. Space Sci., 56, 1748 Sauerbrey, G. 1959, Z. Physik, 155, 206

Seperuelo Duarte, E., Domaracka, A., Boduch, P., et al. 2010, A\&A, 512, A71

Span, R., \& Wagner, W. 1996, J. Phys. Chem. Ref. Data, 25, 6

Stephenson, R. M., \& Malanowski, S. 1987, Handbook of the Thermodynamics of Organic compounds (New York: Elsevier)

Stull, D. R. 1947, Ind. Eng. Chem., 39, 517

van Broekhuizen, F. A. 2005, Thesis, The University of Leiden, The Netherlands

Viti, S., Collings, M. P., Dever, J. W., McCoustra, M. R. S., \& Williams, D. A 2004, MNRAS, 354, 1141

Whittet, D. C. B., \& Duley, W. W. 1991, A\&A, 2, 167

Whittet, D. C. B., Schutte, W. A., Tielens, A. G. G. M., et al. 1996, A\&A, 315, L357

Xiang, H. W. 2004, J. Phys. Chem. Ref. Data, 33, 4 